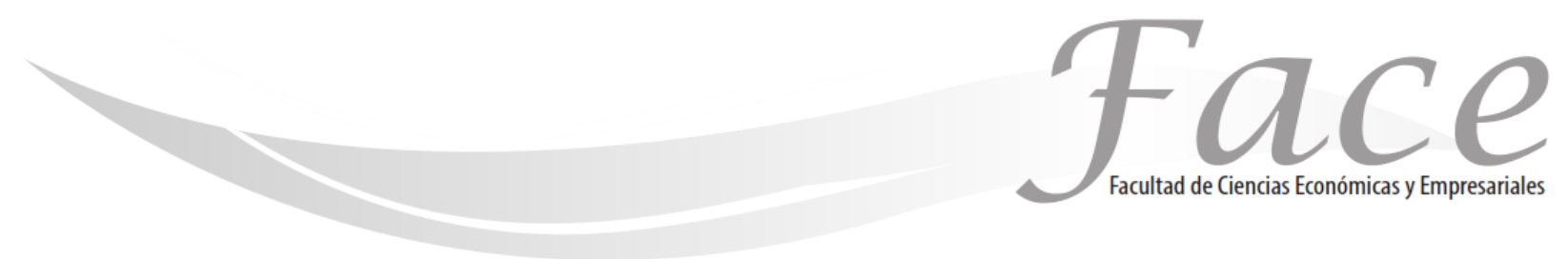

ISSN Impreso: 1794-9920

ISSN Electrónico: 2500-9338

Volumen $18-\mathrm{N}^{\circ} 2$

Año 2018

Págs. 15 - 23

\title{
LIDERAZGO REFORMADOR: COMPORTAMIENTO HUMANO EVOLUTIVO HACIA LA CONCIENCIACIÓN DE LA GERENCIA EN LA INDUSTRIA FARMACÉUTICA
}

\author{
Ramiro A. Buitrago A * \\ Enlace ORCID: https://orcid.org/0000-0002-8563-7662
}

Fecha de Recepción: 4 de Junio 2018

Fecha de Aprobación: 27 de Noviembre 2018

\section{Resumen:}

El presente artículo, es producto de una investigación que contara entre sus propósitos: comprender el liderazgo reformador como el eje que dinamiza la filosofía orgánica, en la misma se llevó a cabo el análisis de los planteamientos hechos por autores como Solórzano (2010), Loaiza (2012), Schaeffer (2002), y Streb (2001), entre otros. Desde un enfoque epistemológico, la investigación contó con un abordaje cualitativo, apoyada en el método de investigación de tradición cualitativa Fenomenológica, con una muestra de treinta y tres (33) sujetos, reducida intencionalmente a un muestreo de cinco (05) sujetos, trabajadores con más de diez años de experiencia en el sector abordado y que durante el desarrollo investigativo laboraban para las unidades de análisis seleccionadas: Farmacia La Coromoto C.A., Farmacia Altagracia C.A. y Farmacia San Benito C.A. En ello se recurrió a la observación no participante y la entrevista en profundidad como técnicas de recolección de información y como instrumentos se empleó la grabadora portátil y el cuaderno de notas. El análisis de los hallazgos se codificó por medio del método de comparación y cuestionamiento continuo, con la finalidad de lograr instruir su categorización, estructuración, triangulación y teorización. Entre los resultados se verificó que con premura se requiere contar con una teoría constructivista del liderazgo reformador con relación a la filosofía orgánica en las organizaciones aliadas a la industria farmacéutica en el municipio Miranda del estado Zulia, Venezuela. Se concluyó que el liderazgo reformador constituye un hecho sociocultural que rebasa el planteamiento del proceso complejo, cuyo desarrollo es cíclico y simbiótico, donde el propósito es la representación de las masas y la reforma de arquetipos o paradigmas establecidos. Se recomienda ejecutar una teoría constructivista en los procesos internos con lugar en el contexto industrial farmacéutico, aún más, en cualquier otro donde la misma pueda robustecer los fenómenos que allí se originen.

Palabras clave: liderazgo reformador, humanización, industria farmacéutica.

\footnotetext{
*Artículo derivado de la investigación intitulada "Liderazgo reformador como eje dinamizador de la filosofía orgánica en organizaciones farmacéuticas" * * Abogado egresado de La Universidad del Zulia (LUZ), Magister en Gerencia de Recursos Humanos / Doctor en Ciencias Gerenciales / Diplomado en Ciencias Forenses y Criminalísticas / Diplomado en el Procedimiento Penal Venezolano egresado de la Universidad Privada Dr. Rafael Belloso Chacín (URBE)/ Abogado IV para la Alcaldía Bolivariana del Municipio Miranda - Edo. Zulia - Venezuela/ Investigador Independiente. Correo: dr.buitrago86@gmail.com
} 


\title{
THE REFORMER LEADERSHIP: EVOLUTIONARY HUMAN BEHAVIOR TOWARDS THE AWARENESS OF MANAGEMENT IN THE PHARMACEUTICAL INDUSTRY
}

\begin{abstract}
:
The present article is the product of an investigation that will have among its purposes: understanding the reforming leadership as the axis that dynamizes the organic philosophy, in it the analysis of the approaches made by authors such as Solórzano (2010), Loaiza (2012), Schaeffer (2002), and Streb (2001), among others. From an epistemological approach, the research had a qualitative approach, based on the research method of qualitative phenomenological tradition, with a sample of thirty-three (33) subjects, intentionally reduced to a sample of five (05) subjects, workers with more than ten years of experience in the sector addressed and that during the research development worked for the selected analysis units: La Coromoto C.A., Pharmacy, Altagracia C.A. Pharmacy and San Benito Pharmacy C.A. In this, non-participant observation and in-depth interviews were used as information gathering techniques and as instruments the portable recorder and the notebook were used. The analysis of the findings was codified by means of the method of comparison and continuous questioning, with the purpose of achieving instructing its categorization, structuring, triangulation and theorization. Among the results it was verified that in a hurry a constructivist theory of the reforming leadership is required in relation to the organic philosophy in the organizations allied to the pharmaceutical industry in the Miranda municipality of Zulia State, Venezuela. It was concluded that the reforming leadership constitutes a sociocultural fact that goes beyond the complex process approach, whose development is cyclical and symbiotic, where the purpose is the representation of the masses and the reform of established archetypes or paradigms. It is recommended to execute a constructivist theory in the internal processes with place in the pharmaceutical industry context, even more, in any other where it can strengthen the phenomena that originate there
\end{abstract}

Keywords: Reforming Leadership, Humanization, Pharmaceutical Industry.

\section{O REFORMADOR IDERAZGO: COMPORTAMENTO HUMANO EVOLUCIONÁRIO PARA A CONSCIENTIZAÇÃO DA GESTÃO NA INDÚSTRIA FARMACÊUTICA}

\author{
Resumo:
}

0 presente artigo é o produto de uma investigação que terá entre seus objetivos: compreender a liderança reformista como 0 eixo que dinamiza a filosofia orgânica, nela a análise das abordagens feitas por autores como Solórzano (2010), Loaiza (2012), Schaeffer (2002) e Streb (2001), entre outros. A partir de uma abordagem epistemológica, a pesquisa teve uma abordagem qualitativa, baseada no método de pesquisa da tradição fenomenológica qualitativa, com uma amostra de trinta e três (33) sujeitos, intencionalmente reduzida a uma amostra de cinco (05) sujeitos, trabalhadores com mais de dez anos de experiência no setor abordado e que durante 0 desenvolvimento da pesquisa funcionou para as unidades de análise selecionadas: Farmacia Coromoto CA, Farmacia Altagracia CA e San Benito Pharmacy C.A. Nesta, a observação não participante e entrevistas em profundidade foram utilizadas como técnicas de coleta de informações e, como instrumentos, o gravador portátil e o notebook foram utilizados. Análise dos resultados foi codificada pelo método de comparação e questionamento contínuo, a fim de alcançar instruir sua categorização, estruturação, triangulação e teorização. Entre os resultados constatou-se que, com pressa, é necessária uma teoria construtivista da liderança reformadora em relação à filosofia orgânica nas organizações aliadas à indústria farmacêutica no município de Miranda, estado de Zulia, Venezuela. Concluiu-se que a liderança reformista constitui um fato sociocultural que vai além da abordagem do processo complexo, cujo desenvolvimento é cíclico e simbiótico, onde 0 objetivo é a representação das massas e a reforma dos arquétipos ou paradigmas estabelecidos. Recomenda-se a execução de uma teoria construtivista nos processos internos com lugar no contexto industrial farmacêutico, ainda mais, em qualquer outro onde possa fortalecer os fenômenos que aí se originam.

Palavras-chave: Reforma da Liderança, Humanização, Indústria Farmacêutica. 


\section{INTRODUCCIÓN:}

\section{Liderazgo Reformador}

Por muchos años la industria farmacéutica persigue el desarrollo de la humanización en sus estructuras internas desde el enfoque estratégico, gerencial e incluso operativo, con el interés de satisfacer necesidades, atender intereses, dar respuesta a las interrogantes que emerjan de su interior, cuestiones como la consciencia, identificación, satisfacción, han sido, quizás de las aristas más investigadas en el acontecer industrial actual. Ahora bien, en opinión de autores como Buitrago (2017), el liderazgo es un proceso que envuelve serenidad, energía y fuerza, para tomar determinaciones precisas, como la destreza de ejecutarlas de una forma efectiva en la consecución de las metas propuestas, bien de forma personal, como aquellas que engloban las del entorno organizacional.

Para Buitrago (2017), al reconocer el liderazgo como fenómeno atinente al comportamiento humano y organizacional contextualizándole en la realidad moderna, se persigue inspirar a los colaboradores identificados con la industria farmacéutica. En ello el triunfo del liderazgo reformador no estriba únicamente en la influencia que ejerce sobre sus seguidores, al mismo tiempo aborda, desarrolla en su ejercicio, el impulso de distintos fenómenos psicológicos, culturales, sociales y hasta personales. Dado lo anterior, el desarrollo de este artículo se ha enfocado en el análisis específico del liderazgo reformador, pero de los gerentes y líderes del rubro farmacéutico, de tal forma, que al ejercer influencia sobre los trabajadores en tales escenarios.

En opinión de Buitrago (2016), no son otros si no los líderes quienes logran con efectividad y sostenibilidad modificar los patrones de comportamiento en el interior de aquellos equipos de trabajo considerados tradicionales, así como son orientadores, también logran ser inspiradores en el proceso de liderar, puesto que quienes forman parte de sus filas, perciben la necesidad que se origina en su interior por identificarse con las aspiraciones del reformador, lo cual resulta ser literal y metafóricamente muy efectivo.

Ahora bien, con el desarrollo de las nuevas tecnologías de la información y comunicación, el auge de las plataformas digitales, desarrollo de la web semántica, y el impacto que la globalización apoyada en el capitalismo hegemónico han ejercido gran influencia en las estructuras orgánica internas de la industria farmacéutica moderna.
Dado lo anterior, en los últimos años diferentes organizaciones aliadas al sector indicado con el interés de humanizar los procesos que en ellas tuviesen lugar enfocaron sus estrategias y políticas de acción en el holismo, la interdisciplinariedad, multidisciplinariedad, así como la transdisciplinariedad.

Para Buitrago (2015), en el estudio del comportamiento humano resulta interesante con atención a lo antes plateado exponer que dada la necesidad al interior de las organizaciones por procurar ajustarse a las transformaciones del orbe moderno los cánones rigurosos, los criterios exiguos y políticas estáticas han quedado atrás, siendo superadas por el protagonismo de la persona muy por encima de lo que en épocas pasadas era lo que imperaba, la producción en masa, la ganancia monetaria, es el momento de las emociones en las organizaciones, los líderes reformadores, entienden, que negarse a estas, es rechazar su propia humanidad, desarrollo, florecimiento, ascenso.

Según Buitrago (2016), la imagen del dirigente infalible, que ha llegado a la cima sin tomar en cuenta las circunstancias de su entorno o las emociones de sus colaboradores, miembros de la organización, trabajadores, seguidores, ya no existe. El crecimiento, el interés por continuar evolucionando, no deja de existir, sin embargo entiende que quienes le rodean, también tienen influencia en dicha conquista, así también, son un factor determinante en el progreso de la organización de la que hacen parte, en ello radica el interés de las ciencias sociales por redescubrir cada día un fenómeno emergente que permita a las organizaciones ofrecer a los trabajadores alternativas que les permita evolucionar y ser más felices.

De acuerdo con Buitrago (2017), el liderazgo reformador, podría conseguir de manera asertiva un cambio comportamental en las personas, propiciando una comunicación clara, ecuánime, alternando relaciones interpersonales entre los trabajadores que pudieran resultar mucho más empáticas e inspiradoras a razón de su humanidad en el ejercicio del poder, alcanzando la mejor contestación por parte de cada colaborador desde la alta gerencia, hasta los trabajadores operativos, todo lo anterior, se muestra como aquello que un reformador es capaz de lograr, por cuanto le resulta fácil en la medida que entiende que de esa forma obtiene evolución como líder, al orquestar los hilos en las organizaciones que gestione. 
En este orden de ideas, el liderazgo reformador, como adalid de la evolución en los entornos corporativos u organizacionales, tiende a mostrar influencia en diversos escenarios del sector privado, entre los que se cuenta a la industria farmacéutica, así también puede desarrollar objetivos simples a la vez que complejos, por ello, en este artículo se considera importante analizar en qué forma estas cualidades del perfil reformador, ejerciendo su poder al interior del rubro abordado logra antes que cualquier otro mejores resultados, desarrollando capacidades propias. Dada la consideración de los autores sobre su potencial al influenciar el comportamiento de los miembros en las organizaciones, siendo ello objeto de interés, por cuanto dicho comportamiento puede estar vinculado con el logro de objetivos fundamentales.

\section{MARCO TEÓRICO:}

\section{Liderazgo Reformador}

Es necesario que el liderazgo reformador consiga trasladar a los diferentes actores involucrados en los escenarios donde ejerza la máxima expresión de su poder una sensación de necesidad y urgencia por cambiar lo establecido. Para ello, resulta de gran importancia contar con la sensibilidad humana suficiente que le permita detectar los elementos de presión externa e interna, aprovechando su habilidad para integrarlos en un discurso de innovación, logrando comunicar este último de manera eficaz. Cabe destacar que la viabilidad de las reformas exige construir alianzas, donde se apoyen las iniciativas de cambio, tal cual reza La Carta Iberoamericana de la función Pública (2005).

En ese mismo sentido, Schaeffer (2002), expone que el liderazgo es algo más que tener mano dura, amén de ello conviene definir las características del cambio, teniendo en cuenta las exigencias y extravagancias de terceros colaboradores en su gestión al interior de las organizaciones, del sector industrial donde ejerza tal rol e incluso del mundo en general, en otras palabras, liderar no es un acto, es un viaje, no existe línea divisoria entre un estilo de liderazgo u otro, un líder autocrático, en ocasiones puede ser participativo, un reformador a veces tendrá que actuar como un autocrático.

De acuerdo con Loaiza (2012), pocas personas nacen con el don de ser líderes, ser líder puede aprenderse tanto como desarrollarse. Todos tienen la posibilidad de hacerlo una opción en su vida. El apego a las estructuras clásicas jerárquicas del liderazgo tradicional en las organizaciones estimula la sumisión. Cualquier integrante de un equipo puede asumir la función de líder. Todo líder necesita de unos seguidores, camina adelante, guía. Por su parte Buitrago, Hernández y Hernández (2017), convienen en que los reformadores expresan sus aspiraciones 0 propósitos comunicando, expresando aquello que está bien y como podría ser mejor, entienden que solo siendo claros en sus expectativas pueden lograr la mejora de los resultados alcanzados por quienes forman parte de su equipo.

Continúa Solórzano (2010), a propósito del liderazgo reformador, el líder siempre está presente. No es un dirigente de estilo autoritario, que determina todas las actividades y la organización del grupo, manteniéndose al margen de toda la participación activa de dicho conjunto, tampoco se trata de un líder pasivo que solo proporciona ayuda e información cuando se la piden. El líder, tiene que dar el ejemplo, debe ser democrático, someter a las decisiones al grupo. El primero que las cumple.

Para autores como Streb (2001), este liderazgo, tiene la capacidad de influir en el consenso necesario para efectuar cualquier tipo de reforma institucional, suelen manifestar una confianza total en su propio criterio, y su capacidad, ostentando una meta idealizada que proponga un futuro mejor para el statu quo, cuentan también con la capacidad para exponer, aclarar su visión en términos comprensibles para otras personas.

Igualmente Schaeffer (2002), expone que ser un líder reformador, es gratificante, pero también comporta sus peligros. En la gerencia estratégica, el líder reformador, puede introducir cambios a gran escala, consolidando las organizaciones, luchando por la supervivencia corporativa, conducir la compañía al éxito en el mercado, o mejorar la industria en la que pertenezca, las exigencias del mercado modelan la forma en que se gestiona el liderazgo, prestando atención a los procesos, alineando los equipos para que estén tan dedicados a alcanzar tal como lo estaría el líder reformador, sería entonces posible, crear algo que duraría más que cualquier escultura tallada en un bloque de hielo.

Continua Streb (2001), el reformador, en su apariencia debe ser la de un agente de cambio radical, y no como individuos que vienen a mantener el orden vigente, por otra parte, vale destacar que estos líderes son capaces de hacer evaluaciones realistas de las restricciones 
ambientales, y de los recursos necesarios para efectuar el cambio, y adaptar sus conductas de acuerdo a estos. A este respecto Solórzano (2010), expone que tiene que basarse en ideas claras, ideas nuevas, revolucionarias, que impliquen los intereses del conjunto de la sociedad nacional e internacional. Y está claro que los intereses mundiales están en la multipolaridad, varios centros de poder global, en ningún caso se plantea un solo centro de poder.

Por último Loaiza (2012), apunta que el líder comprometido con los cambios es un modelo a seguir. Para que el proceso de liderar tenga repercusión, éxito, sostenibilidad conviene hacerlo con principios éticos, reconociendo en la gente en sus habilidades y capacidades, siendo hábil en las relaciones interpersonales y demostrando coraje. Para Streb (2001), los reformadores cuentan con convicciones firmes en torno a su visión, denotando un fuerte compromiso, manifestando enérgicos deseos de asumir riesgos personales, su comportamiento suele ser novedoso, según el entorno en que ejerza el desenvolvimiento de su personalidad, fuera de lo convencional y quizás contrario a las normas, paradójicamente, esta certeza en sus actos generan confianza y admiración entre sus seguidores.

\section{Liderazgo de la Reforma en una Época de Cambio}

En opinión de autores como Becerra (2010), los procesos complejos que se desarrollan en las organizaciones modernas, llevan consigo la incertidumbre, inestabilidad e incapacidad de lograr certeza. La comprensión de la complejidad, requiere de un cambio profundo de la forma de concebir la realidad. En otras palabras, pensar en la sociedad como un todo organizado, y organizador, que retroactúa para formar a los individuos, a través del lenguaje y la educación. Para Becerra y Sánchez (2011), la dinámica del conocimiento presenta un reto, cada organización tiene que construir la dirección del cambio dentro de su estructura, debido a que cada una, tiene que prepararse para abandonar todo lo que hace, es decir, requiere incorporar a la vida, diaria la autosuperacion continua.

Para Zamora y Poriet (2008), convienen en que las organizaciones están enmarcadas en un contexto de continuos avances tecnológicos, inmersas en una economía global, con altos niveles de competitividad, fluctuación, estrategias de orientación, servicio al cliente y énfasis en el capital humano. De modo tal, que exigen cambios de índole estructural, principalmente de prácticas orientadas a la participación y desarrollo de alianzas. Autores como Schaeffer (2002), sostienen que un reformador, demuestra lo que es posible, desafía los convenios e intenta a toda costa hacer del mundo un lugar mejor. Para el líder reformador, su misión es cambiar la industria en la que es participe, es una cuestión de cambio en la reputación que pueda ostentar el sector del que sea parte.

Continua exponiendo Solórzano (2010), con respeto al líder reformador no se queda solo en la renovación, sino que en realidad se trata de revolución, transformación, porque si no, ¿qué sentido tendría para la política o para lo que competa al aspecto militar, social, cultural? -Poco-. De lo que se está hablando, es de ser pioneros, de idea nueva, mensaje nuevo. Así pues, Jesús, fue vanguardia, un líder reformador, por la acción, por la idea. Para Becerra y Sánchez (2011), en una organización inteligente, los líderes son diseñadores, administradores y maestros, con ello ofrecen a sus colaboradores oportunidades donde desarrollar continuamente su aptitud para comprender la complejidad, clarificar la visión y mejorar los modelos mentales compartidos.

Para Becerra (2010), una organización es producto de la manera como la gente piensa, actúa. Cambiar una organización significa darles oportunidad a las personas para que puedan revisar, reflexionar sobre su manera de actuar o pensar, ya que ninguna persona por si sola puede cambiar sus actitudes. Cuando a estas se les da la oportunidad de participar en tal dinámica, llegan a desarrollar una capacidad perdurable para el cambio. Preparar el cambio equivale a crecer, y crecer equivale a estar satisfecho. Igualmente, Becerra y Sánchez (2011), a propósito del liderazgo reformador, exponen, que estos nuevos líderes, deben mostrar directrices organizacionales innovadoras, más específicamente, estrategias para adaptarse a las situaciones de cambio y una visión holística favoreciendo las transformaciones.

Por su parte Gómez de Souza y otros (2014), sostienen, que en las organizaciones actuales, el liderazgo de la gestión se caracteriza por ideas cimentadas en la innovación y valoración de la originalidad, que resaltan su sentido del cambio. Estas características, son más comúnmente observadas en las corporaciones que se destacan por su liderazgo reformador, con carácter 
carismático, patriarcal. Según Moreno, Granda y Camisón (2008), el liderazgo reformador, no solo reviste las características de emprendimiento, innovación, comunicación, confianza, respeto, es también responsable y consciente del conocimiento que ostenta, de la información que en algunos momentos pudiera echar en falta.

A propósito Solórzano (2010), continúa planteando que, en la sociedad moderna, el líder está obligado a ser un constante agente de cambio, tiene la obligación de estar cada vez mejor preparado para llevar adelante el cometido. El reto cotidiano es conducir el cambio, influir en su rumbo, con verdadera capacidad organizativa. Por su parte Becerra (2010), apunta, los nuevos líderes dentro de una organización deben mostrar directrices organizacionales, gerenciales innovadoras, más específicamente estratégicas, para adaptarse a las situaciones de cambio, y una visión que, compartida con todos los actores, conduzca a que estos actúen con iniciativa propia, favoreciendo las transformaciones, necesarias que requiere la organización en pro de mantener su vigencia en el mercado.

Según Noriega (2008), el nuevo milenio ha transformado las prácticas de las organizaciones y con ello las habilidades, características que el actual entorno empresarial demanda de los líderes. El líder de hoy debe poseer un perfil muy distinto del de hace varias décadas, cuyo patrón se ajustaba en mayor medida al control, la supervisión. A los líderes del siglo XXI se les exige una preparación diferente, para poder atender las necesidades de las empresas modernas. Según Becerra (2010), lo que define si una persona está funcionando dentro de ese sistema dinámico llamado crecimiento, son sus competencias personales, el uso que hace de las mismas. Si las usa para ver el mundo desde diferentes enfoques permanentemente, aumenta al mismo tiempo su repertorio de competencias.

Continúan Zamora y Poriet (2008), planteando que de este modo se observa un cambio radical en el liderazgo de este momento, y el que se ha venido utilizando para dar respuesta, soluciones a los problemas de épocas precedentes. Romper estos paradigmas de la forma de trabajar en las organizaciones. En este mundo hipercompetitivo, cambiante de inicios del siglo XXI y años subsiguientes, permitirá repotenciar a las organizaciones para que generen nuevas formas de trabajar, nuevas riquezas.

\section{METODOLOGÍA:}

Esta investigación fue desarrollada bajo el paradigma cualitativo, con apoyo al método fenomenológico, donde la observación no participante, la entrevista en profundidad e historias de vida, fueron técnicas aplicadas por el investigador, el cual hizo uso de diversos instrumentos entre los que se cuentan: la grabadora portátil, el cuaderno de notas y las notas sobre notas, para así garantizar confiabilidad y validez en su labor indágativa con el propósito de plantear nuevas perspectivas, amparándose en la descripción, abstracción e interpretación así como también confrontación de los hallazgos obtenidos. Respecto a lo anterior Sabino (2006), plantea las investigaciones descriptivas se proponen conocer grupos homogéneos de fenómenos para establecer una descripción de sus características.

Así entonces, se abordó al liderazgo reformador dinamizador de la filosofía orgánica, como fenómeno universal y complejo con lugar en las organizaciones farmacéuticas del municipio Miranda del estado Zulia, Venezuela, por medio de las técnicas de investigación aplicadas, confrontadas con los hallazgos logrados a través de la revisión bibliográfica documental. Ahora bien, el artículo en cuestión se apoya en los resultados arrojados por el proceso explorativo de documentos escritos, donde diferentes autores establecían bases teóricas resultantes de sus trabajos investigativos. Respecto a los estudios documentales, Sierra (2008), plantea que consisten en el análisis de informaciones con apoyo en documentos, estos se organizan, con el propósito de dar origen al conocimiento nuevo, considerando como referencia, publicaciones arbitradas, válidas para el estudio.

Igualmente, es importante resaltar que el diseño de la investigación de la cual se desprende el presente artículo estuvo dividido en cuatro momentos: el momento I, donde se realizó una indagación profundamente epistemológica en relación a los fenómenos seleccionados, el momento II, tomo en cuenta la revisión de bibliografía relacionada con la temática, luego el momento III, permitió estos fenómenos fuesen contextualizados en las organizaciones farmacéuticas venezolanas, unidades de análisis 
seleccionadas para tal fin, posteriormente, el momento IV, mostró los resultados ofrecidos, luego de un proceso investigativo complejo, hermenéutico y profundo.

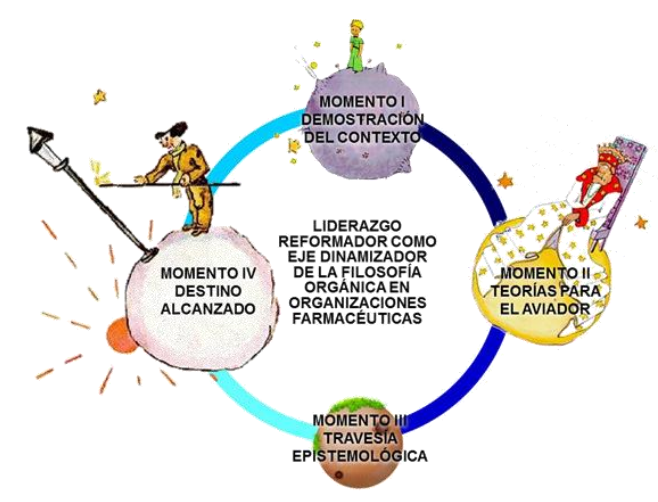

Fuente: Elaboración propia (2016), adaptación del modelo propuesto por Del Canto (2012).

Las organizaciones elegidas para el proceso investigativo, atienden la estructura administrativa orgánica idónea para que cada una de las unidades de análisis fuese lo suficientemente fructífera y relevante con la investigación, así entonces puede inferirse que los trabajadores, gerentes, y adjuntos, pertenecientes a los establecimientos comerciales aliados a la industria farmacéutica seleccionados, contaron con las cualidades y recursos intelectuales suficientes como para ser sujetos de este trabajo, realizado en la ciudad de Los Puertos de Altagracia, localidad del municipio Miranda, estado Zulia, Venezuela. En este contexto, correspondió a tres establecimientos comerciales Farmacia La Coromoto C.A., Farmacia Altagracia C.A. y Farmacia San Benito C.A.

Para ello, se conformó una muestra integrada por treinta y tres (33) trabajadores adscritos a las unidades de análisis seleccionadas, la cual, luego de un proceso de observación consecuente e imparcial, se redujo, considerando disimiles razonamientos investigativos, adecuados al enfoque de este trabajo, contando así con el primer muestreo teórico, mismo que facilitó el alcance de una muestra intencional de cinco (05) sujetos, asociados a las unidades de análisis seleccionadas: Farmacia La Coromoto C.A., Farmacia Altagracia C.A. y Farmacia San Benito C.A., a tales grupos se les hizo parte en tres entrevistas atendiendo la saturación de las categorías emergentes, finalmente con el informante número dos (02), se alcanzó llenar algunos vacíos teóricos resultados del proceso.

Esta investigación, se realizó en lapso comprendido entre septiembre de 2014, hasta julio del 2016, la misma se prolongó por 22 meses, cuestión que atendió las exigencias institucionales respetadas por el investigador.
Desde el momento en que fuera concebida, en ella se tuvo como propósito generar una teoría humanista e innovadora capaz de facilitar el proceso evolutivo de la situación descrita, visualizándose en ello, el progreso organizacional, aspirando solucionar una situación problemática relacionada estrechamente con las necesidades humanas, vinculándole fundamentalmente al sector privado.

\section{RESULTADOS Y CONCLUSIONES:}

Es importante destacar que el liderazgo reformador como proceso, se desarrolla en escenarios donde el comportamiento humano es mucho más dúctil, es decir, aquellos entornos donde las personas puedan desenvolver de forma libre su personalidad, puesto que quienes se reconocen como líderes reformadores, ejercen su manera de ser como mejor saben hacerlo, liderando. Con referencia a lo anterior, en esta investigación se propuso al liderazgo reformador como el eje dinamizador de los procesos gerenciales con lugar al interior de la industria farmacéutica como el adalid que permitiría la ductilidad de fenómenos propios con origen en tales escenarios, todo lo cual habilita desarrollar perfiles de gestión mucho más eficientes, eficaces, efectivos.

Ahora bien, advirtiendo las disimiles formas en que el líder reformador, muestra para cumplir con sus propósitos como adalid en una época de cambio, permitiendo a sus seguidores brillar por sí mismos, destacarse, ejerciendo un liderazgo integrador, humano, reformador de los preceptos de otrora. En ese sentido, tal rol no puede ser ejercido por cualquiera, más cualquiera, puede ser líder, en efecto, no todo el que quiera serlo, tendrá lo suficiente para poder estar al frente de tales procesos, más de cualquier escenario social, podría emerger un líder reformador, que cumpla con las necesidades, aspiraciones de aquellos que manifiesten la intención de seguirle, así como el interés de apoyar sus ambiciones a la voluntad de otro, que ostente las habilidades, destrezas necesarias.

Por ello se plantea que en tales organizaciones puede ser ejercido el liderazgo reformador como un proceso, sí, pero también más allá de ello, un fenómeno sociocultural donde hombres y mujeres intercambian su apreciación sobre cuestiones profundas, complejas, de las que dependa, sin ánimos de dramatizar la situación, su felicidad. En ese sentido, se percibe como fenómenos como el liderazgo reformador contextualizado en de forma tan interesante como profunda, permite al investigador encontrar una oportunidad hacia la generación de una teoría constructivista, cuyo interés no es otro que, el desarrollar conocimiento nuevo que pueda ser aprovechado por él mismo, así como por aquellos que de 
alguna forma u otra estén interesados en la humanización de la industria farmacéutica.

Finalmente, puede plantearse que tanto el liderazgo como hecho sociocultural con relación a otros fenómenos atinentes al comportamiento humano pueden darse de manera simultánea, según las formas orientadoras en el accionar del líder, con función a las características de la organización donde estos tengan lugar. Por ello, se expone que el mismo cuenta con elementos sui generis que resultan esenciales y en ausencia de los cuales no podría hablarse 0 dar constancia de su presencia. Recomendándose así que partiendo de investigaciones como esta se generen muchas otras hacia el abordaje de tal fenómeno desarrollando así contenidos que apunten a la existencia de factores muy específicos para poder auscultar si se está en presencia de un líder reformador.

\section{REFERENCIAS:}

Becerra, G. (2010). El liderazgo y su rol en las organizaciones transcomplejas. Visión Gerencial. (1). Pp.: 30 - 42. Mérida. Venezuela.

Becerra, M. y Sánchez, L. (2011). El liderazgo en las organizaciones inteligentes. Revista científica digital del centro de investigación y estudios $\begin{array}{llll}\text { gerenciales. } \quad 1 & \text { (4). } \quad \text { Pp.: } & \text { 61-71. }\end{array}$ Barquisimeto. Venezuela.

Buitrago, R. y Portillo, P. (2014). El poder, las mujeres y su liderazgo ejercido en el sector público. Revista Fórum Humanes. 3(2). Pp. 45-59. Maracaibo. Venezuela.

Buitrago, R. (2015). El liderazgo gerencial femenino, una propuesta a la industria farmacéutica. Revista CICAG. 12(2). Pp. 212-225. Maracaibo. Venezuela.

Buitrago, R. (2016). Liderazgo ético como factor potenciador del marketing social hacia la innovación estratégica en la mercadotecnia moderna. RevistaMarketing Visionario. 5(1). Pp. 72-87. Maracaibo. Venezuela.
Buitrago, R. (2016). Liderazgo reformador como eje dinamizador de la filosofía orgánica en organizaciones farmacéuticas. Universidad Privada Dr. Rafael Belloso Chacín. (Tesis Doctoral no publicada). Maracaibo. Venezuela.

Buitrago, R. (2017). Gestión del conocimiento a través del liderazgo femenino como recurso esencial para la competitividad organizacional. Revista CICAG. 14(2).Pp. 320-337. Maracaibo. Venezuela.

Buitrago, R. (2017). Liderazgo de servicio como factor amplificador del pensamiento estratégico en la gerencia de las organizaciones modernas. RevistaCOEPTUM. 8(2). Pp. 152-172. Maracaibo. Venezuela.

Buitrago, R., Hernández, M. y Hernández, P. (2017). Liderazgo resonante y su efecto dinamizador en la negociación ante conflictos organizacionales. En Desarrollo Gerencial Revista de la Facultad de Ciencias EconómicasAdministrativas y Contables de la Universidad Simón Bolívar-Colombia, 9(1), Pp.: 97-111. Barranquilla. Colombia.

Del Canto, E. (2012). Investigación y métodos cualitativos: un abordaje teóricodesde un nuevo paradigma. Revista ciencias de la educación. 22(40). Pp.: 181-199. Valencia. Venezuela.

Gómez de Souza, A. Barboza, M., Kovas, M. y de Brito, M. (2014). Gestión hotelera. El caso Brasileño de la red de Pontes Hoteis \& Resorts bajo el modelo deorganización multidimensional reflexivo. Estudios y perspectivas en turismo. Volumen 32 (2014) pp 768 - 785. Brasil.

La Carta Iberoamericana de la función Pública. (2005). Foro Iberoamericano: Revitalización de la Administración Pública. Estrategias para lalmplementación de la Carta Iberoamericana de la Función Pública.México. (2005). 
Loaiza, A. (2012). Reconocimiento humano como factor potenciador del liderazgo en universidades de gestión privada. Universidad Privada Dr. Rafael Belloso Chacín, (Tesis Doctoral no publicada). Maracaibo. Venezuela.

Moreno, C., Granda, G. y Camisón, C. (2008). El modelo de empresa del siglo XXI: Hacia una estrategia competitiva y sostenible. Ética empresarial liderazgosresponsables, Colección Biblioteca Ciencias Sociales. Primera edición. Enero 2008. Grupo editorial Cinca, S. A. Madrid. España.

Noriega, G. (2008). La importancia del liderazgo en las organizaciones. Ensayos. Temas de ciencia y tecnología. Instituto de ciencias Sociales y Humanidades.Universidad tecnológica de la mixteca. Vol. 12. n 36. 2008. México.

Sabino, C. (2006). Los caminos de la ciencia: una introducción al método científico, Buenos Aires: Lumen-Humanitas, 2006. Buenos Aires. Argentina.

Schaeffer, L. (2002). El viaje hacia el liderazgo. Editorial Deusto. Primera Edición.L.A. California. EUA. 2002.

Sierra, R. (2010). El método investigativo. Editorial Suramérica. Caracas, Venezuela

Solórzano, C. (2010). Liderazgo, Socialismo. Fondo editorial IPASME. Depósito Legal:

IF65120093204671. ISBN: 978-980-401-020-0. Caracas. Venezuela. 2010.

Streb, J. (2001). Liderazgo político reformador estratégico. Pseudonimo: NovoOrodo Seclorum. Universidad del Cema. Buenos Aires. Argentina.

Zamora, A. y Poriet, Y. (2008). Papel de los líderes y nuevas tendencias del liderazgo en el siglo XXI. Revista faces. Volumen xvii. $n^{0} 1$. Escuela deRelaciones Industriales Universidad de Carabobo. Valencia. Venezuela. 